\title{
La expansión de la cría de camarón en las costas del golfo de Fonseca, Centroamérica, 1985-2011
}

\author{
Rafael Enrique Corrales Andino ${ }^{1}$ \\ Manuel Pérez Gómez, Eduardo Corbelle Rico, Miguel Cordero Souto ${ }^{2}$
}

\section{RESUMEN}

En las últimas décadas, las instalaciones de camaricultura se han convertido en una de las actividades con mayor importancia económica en la costa sur de Honduras. Por la naturaleza de esta actividad y por su ubicación en el golfo de Fonseca, fue declarada área sitio Ramsar desde 1999; además de categorías de áreas protegidas, por el SINAPH, existe preocupación por su influencia en la disminución y degradación del ecosistema manglar, de alto valor ecológico. En este contexto, el principal objetivo de este trabajo es hacer un seguimiento de la superficie ocupada por la camaricultura y ecosistema manglar en el golfo de Fonseca, entre los años 1985 y 2011. Asimismo, se prestó atención a posibles transvases (directos o indirectos), que se habrían producido entre estas dos clases de cubiertas de suelo a lo largo del período estudiado. Para todo esto se emplearon imágenes satelitales (LandSat 5) del periodo comprendido entre 1985 y 2011. Los resultados del análisis indican que el avance de la actividad camaronera (de 2,140 ha en 1985 a 41,434 ha en 2011) ha ocurrido al mismo tiempo que una disminución importante del área de manglar y otros humedales costeros (de 107,167 ha a 78.509 ha en el mismo período). Los cambios o transiciones ocurridas entre cubiertas sugieren que la camaricultura ha ocupado alrededor del $14 \%$ de la superficie del manglar existente al inicio del período. Esta expansión supuso solo cerca de un $39 \%$ de la superficie ocupada por esta actividad, ya que esta habría ocupado preferentemente otros tipos de cubiertas naturales previas.

Palabras clave: mangle, camaricultura, golfo de Fonseca.

\footnotetext{
1 Profesor universitario Departamento de Ciencia y Tecnologías de la Información Geográfica (DCTIG-FACES), UNAH: rcorrales@unah.edu.hn

2 Laboratorio do Territorio (LaboraTe), Universidade de Santiago de Compostela: manuel.perez.gomez@usc.es; eduardo.corbelle@usc.es; miguel.cordero@usc.es
} 


\section{ABSTRACT}

In recent decades, shrimp farming facilities have become one of the most important economic activities on the south coast of Honduras. By the nature of this activity and its location in the Gulf of Fonseca, the area was declared a Ramsar site since 1999, also as a categorie of Protected Areas by the SINAPH, there is concern about its influence on the decline and degradation of mangrove ecosystem, which posses a high ecological value. The main objective of this work is to track the area occupied by shrimp farming and mangrove ecosystem in the Gulf of Fonseca, between the years 1985 and 2011, and attention to possible water transfers (direct or indirect), that would have occurred between these two types of ground coverings throughout the studied period. Satellite images (Landsat 5) of the period between 1985 and 2011 were used. The results of the analysis indicated that the advance of shrimp farming $(2,140$ ha in 1985 to 41,434 ha in 2011) occurred at the same time as a significant decrease in mangrove area and other coastal wetlands $(107,167$ ha to 78,509 ha in the same period). Changes or transitions occurring between coverings suggest that shrimp farming occupied about $14 \%$ of the surface of the existing mangrove at beginning of the period. This expansion accounted for just $39 \%$ of the total area occupied by this activity between 1985 and 2011, since this would preferably held other types of prior natural coverings.

Keywords: mangrove, shrimp farming, gulf of Fonseca. 


\section{INTRODUCCIÓN}

La cría de camarón es una forma de acuicultura que ha aumentado considerablemente su importancia en muchas zonas costeras tropicales y subtropicales desde las últimas décadas del siglo pasado. En algunos casos, este aumento tuvo lugar antes de la existencia de evaluaciones ambientales y mecanismos de control por parte de los gobiernos de los países y, como resultado, las posturas aparecen frecuentemente polarizadas entre la valoración de los beneficios económicos que la actividad reporta y la preocupación por sus consecuencias para el medioambiente 0 para otras actividades económicas de la población local (FAO, 2000).

En particular, el tipo de áreas en las que esta actividad se establece, hace que entre en conflicto con el mantenimiento de la superficie y calidad ambiental de las áreas de manglar y otros hábitats costeros de elevada importancia y fragilidad ecológica (Alonso Pérez y otros, 2003; FAO, 2011). Aun cuando las diferentes instituciones del Estado, así como organizaciones no gubernamentales, promueven la conservación de los humedales del golfo de Fonseca declarándolas como áreas protegidas y manejadas para asegurar su equilibrio ecológico, estas cuentan con esa protección por convenios internacionales, como los sitios Ramsar, para protección de humedales desde la década de 1990 en el golfo de Fonseca (The Ramsar Convention, 2014), pero la reducción del área de manglar es un hecho preocupante en Honduras, al igual que en los países vecinos; según algunas fuentes podría haber afectado a más de tres cuartas partes de la superficie existente hacia 1960 (FAO, 2005). La degradación y, en última instancia, la desaparición de estos ecosistemas supone un problema importante debido a que desaparecen las funciones ambientales como el servir de barrera para las tormentas tropicales, hábitat de numerosas especies, filtro para sólidos en suspensión o fuente de materia orgánica (Alonso Pérez y otros, 2003). Otros potenciales efectos negativos de la cría de camarón se derivan de la recolección de larvas de camarón silvestre o del uso de sustancias químicas para la limpieza de las lagunas de cría, que a menudo acaban en las aguas superficiales cercanas.

\section{DESCRIPCIÓN DEL ÁREA DE ESTUDIO}

El área de estudio total en la que se desarrolló este trabajo comprende el área costera 
del golfo de Fonseca en territorio de Honduras, El Salvador y Nicaragua. Para restringir el área total objeto de análisis a las zonas susceptibles de alojar alguna de las dos cubiertas del suelo de interés en este trabajo (camaricultura y manglares), se utilizó la elevación como criterio de selección. En particular, se empleó la info- rmación disponible en el modelo de elevación digital (DEM) de la Shuttle Radar Topography Mission con paso de malla de 3 segundos de arco (SRTM 3), en la versión publicada por el Consortium for Spatial Information (CGIAR-CSI) con 90 metros de resolución espacial (Jarvis y otros, 2008). Las áreas seleccionadas se corresponden con aquellas situadas entre los 0 a $18 \mathrm{msnm}$ en esta última fuente (ver figura 1).

El área de estudio está rodeada por manglares, por lo cual es propicia para la cría de camarón. Dispone de una llanura topográfica interrumpida por dos porciones montañosas que constituyen los extremos terminales de la cordillera central y de la cadena costera. Esta zona está comprendida dentro de la clasificación climática de sabana tropical caliente, caracterizada por su concentración de lluvias en septiembre y octubre (casi el $40 \%$ de la duración total del año) y temperaturas elevadas (media anual de $\left.27^{\circ} \mathrm{C}\right)$.

Figura 1. Región de interés: golfo de Fonseca (Honduras, Nicaragua y El Salvador)

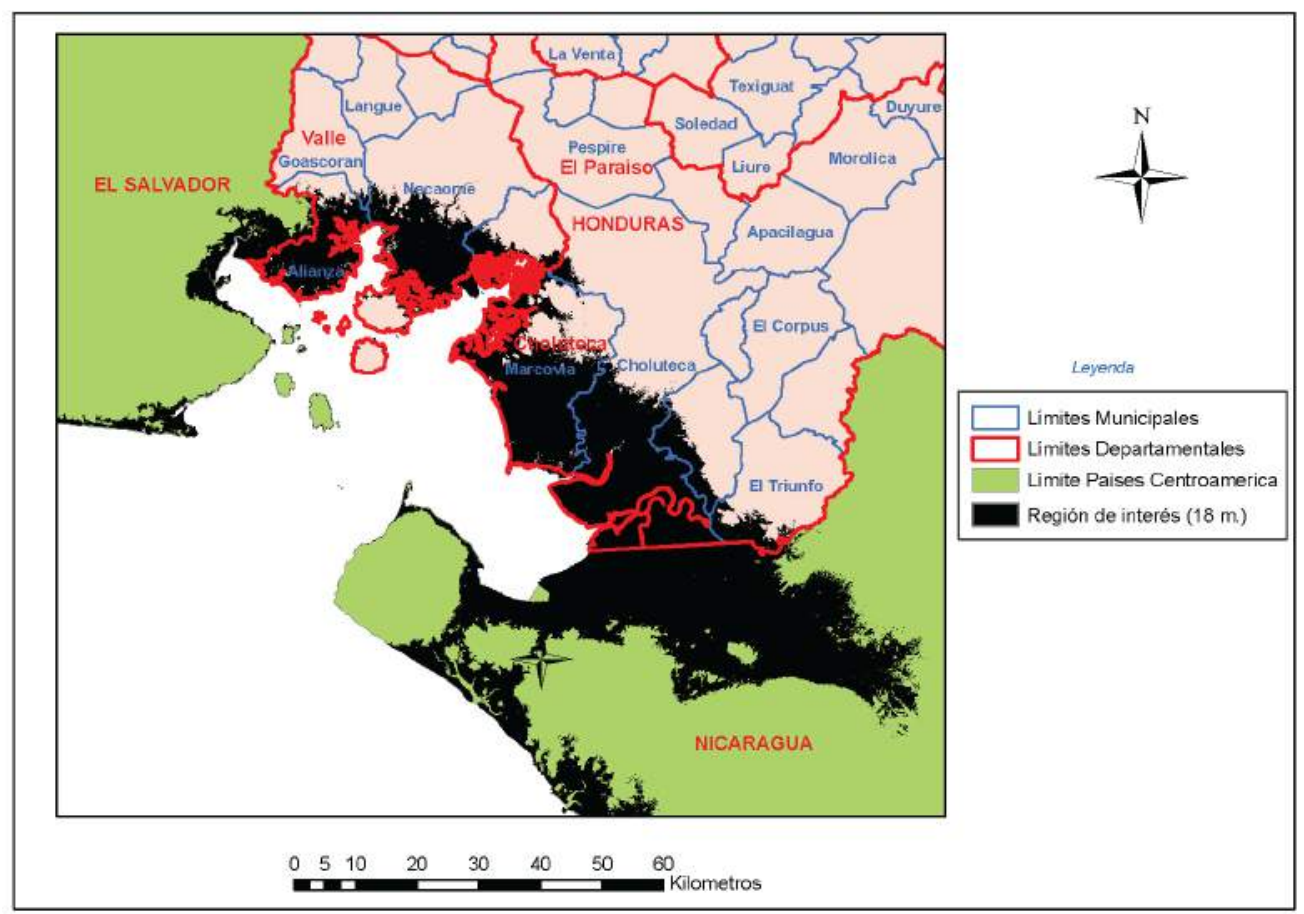




\section{METODOLOGÍA}

A continuación se presentan los materiales (imágenes de satélite), equipo (programas de análisis espacial) y la metodología de análisis multitemporal de cambios, a través de la asignación de clases por cobertura del suelo con imágenes satelitales, desarrolladas por las técnicas de teledetección aplicadas en la investigación

\section{Materiales y equipo}

Las imágenes satelitales empleadas fueron del sensor TM (thematic mapper) a bordo de LandSat 5, obtenidas desde el global visualization viewer del Servicio Geodésico de los Estados Unidos de América (USGS). Se trata de imágenes con un nivel de corrección 1, es decir, con una corrección radiométrica inicial que permite eliminar errores de toma del sensor y una corrección geométrica basada en puntos de control tomados sobre el terreno. La resolución espacial de las bandas es de $30 \mathrm{~m} / \mathrm{pixel}$, excepto en el caso de la banda 6 (120 m/pixel). Cada imagen cubre un área de $185 \times 185 \mathrm{~km}$. Para cubrir toda el área de estudio fue necesario emplear imágenes correspondientes a los cuadrantes LandSat p17r51 y p18r51.

El criterio para la selección de imágenes consistió en identificar preferiblemente dos imágenes libres de cobertura de nubes para cada año, siempre que fuese posible, dentro del período de estudio 1985-2011. En total se obtuvieron 105 escenas, para todos los años excepto, para 1988, 1992, 1995, 2003 a 2006 (incluidos) y 2008.

Aunque hubieran sido de gran utilidad en el trabajo, se descartó utilizar las imágenes producidas por el sensor ETM+ a bordo de LandSat 7, debido al efecto de bandeado que caracteriza a estas imágenes como consecuencia de la avería del dispositivo scan line corrector (SLC) en 2003.

Además de la recopilación de imágenes de satélite, se descargó cartografía temática y vectorial de la página oficial del Sistema Nacional de Información Territorial de Honduras (SINIT), para poder identificar los distintos espacios del golfo de Fonseca (límites administrativos, hidrografía y clima).

Como herramienta de procesamiento de las imágenes de satélite se utilizaron los sistemas de información geográfica libre GRASS (GRASS Development Team, 2012), ya que dispone de algoritmos de clasificación y la interfaz gráfica de QGIS para 
la visualización de los resultados de la clasificación y posterior procesado de información (Bouman y Shapiro, 1994).

\section{Método}

1. Adquisición de imágenes: el conjunto de las imágenes recopiladas fue importado y procesado en GRASS con la ayuda de ficheros de proceso por lotes (scripts).

2. Correcciones radiométricas: cada una de las imágenes fue sometida a un proceso de corrección radiométrica (Riaño y otros, 2000) para transformar los niveles digitales (ND) a parámetros físicos de reflectancia a tope de atmósfera (top-of-atmosphere reflectance) empleando el módulo i.landsat.toar y el fichero de metadatos: (MTL) incluido con cada imagen.

$$
\rho_{\text {TOA }}=\frac{\pi L_{\text {sat }} d^{2}}{\left(E_{0} \cos \theta_{z}\right)}
$$

(MTL) incluido con cada imagen

Lsat $=$ radiancia total medida por el satélite a tope de atmósfera $(L s a t=D N \cdot$ gain + offset $)$ $\mathrm{d}^{2}=$ distancia tierra sol en unidades astronómicas, y $\mathbf{d}$ se calcula como:

$d=1-0.0167 \cos (2 \pi$ (día juliano -3$) / 365)$

$\mathrm{E}_{0}=$ irradiancia solar espectral a tope de atmósfera (watts $/ \mathrm{m}^{2}{ }^{2} \mu \mathrm{m}$ )

$\theta_{z}=$ ángulo cenital solar

3. Clasificación de imágenes: aunque el proceso de corrección radiométrica permitió acercar los valores de las imágenes correspondientes a los dos sectores 0 cuadrantes LandSat, sería necesario para poder procesar las dos imágenes contiguas en un solo paso (mosaico), que se realizase algún proceso de ecualización. Las variaciones en las fechas de adquisición de cada par de imágenes contiguas presentaban siempre una considerable diferencia, hasta el punto de coincidir a menudo con las fases antes y después del llenado de las lagunas de camaricultura en uno y otro cuadrante; razón por la cual se realizó una clasificación automática a las imágenes de cada cuadrante por separado. Entre los procesos de clasificación automática o método de clasificación no supervisado (Schowengerdt, R.A., 2007), uno de los más difundidos es el K- medias, que se define dado un conjunto de observaciones (x1, x2, .., xn), en donde cada observación es un vector real de d dimensiones, k-medias construye una partición de las observaciones en $k$ conjuntos $(k=n) S=\{S 1, S 2, \ldots, S k\}$ : 


$$
\underset{\mathbf{S}}{\arg \min } \sum_{i=1}^{k} \sum_{\mathbf{x}_{j} \in S_{i}}\left\|\mathbf{x}_{j}-\boldsymbol{\mu}_{i}\right\|^{2}
$$

donde $\boldsymbol{\mu}_{i}$ es la media de puntos en $S_{i}$

Cuando las asignaciones ya no cambian, se considera que el algoritmo ha convergido; esto significa que las clases seleccionadas se han ajustado a un número definido sin más variaciones, dándose la convergencia. Para el proceso de clasificación se utilizó el módulo i.cluster disponible en GRASS, una variante del algoritmo de clasificación k-medias. Se trata de un proceso de clasificación no supervisada cuya única intervención del operador concierne al establecimiento del número de clases objetivo. La diferencia del método respecto del algoritmo k-medias, estriba en que i.cluster utiliza aquel para clasificar una muestra de la imagen original, que a su vez es empleada después en un proceso de asignación de clase a todos los píxeles de la imagen por máxima verosimilitud. El número de clases demandado en la clasificación fue de 12.

4. Filtros o kernel: el resultado de la clasificación fue sometido en cada caso a un filtro de moda de tamaño de ventana $5 \times 5$, para reducir la presencia del efecto de "sal y pimienta" (Chuvieco, 2008), debido a la mezcla íntima de clases diferentes en píxeles vecinos.

5. Transformaciones y depuraciones ráster a vector: cada uno de los resultados fue transformado a formato vectorial y depurado manualmente. El proceso de depuración manual consistió en la identificación de las clases originadas en la clasificación automática que se corresponden con alguna de las categorías de interés en el trabajo y la eliminación manual de los errores groseros que pudieran estar presentes. Esta última verificación se realizó utilizando como referencia la imagen original de cada año. Finalmente, el proceso termina con la unión de los ficheros vectoriales correspondientes a un mismo año y dos cuadrantes distintos en un único archivo. Para reducir las discrepancias entre uno y otro lado de la línea de unión entre ambos cuadrantes, se seleccionó manualmente una línea de unión, dentro de lo posible, cursos de agua. Dado que se encontraban disponibles dos clasificaciones (procedentes de imágenes distintas) para cada año y cuadrante, el proceso de depuración se llevó a cabo sobre aquella que representó mejor la situación del territorio en cada momento. Para poder seleccionar una capa vectorial por año, se utilizó como criterio la identificación diferenciada de las siguientes categorías objetivo (de la clasificación final se eliminaron las zonas de mar y cursos de agua), tal como se muestra en la figura 2: 
a. Lagunas de camaricultura

b. Rhizophora mangle o mangle rojo

c. Otras Sp. de manglar

d. Lagunas de invierno

e. Otros tipos de vegetación (para el resto de clases que no se localizan en las anteriores)

Figura 2. Muestra de graduación de color para cada clase en una imagen determinada

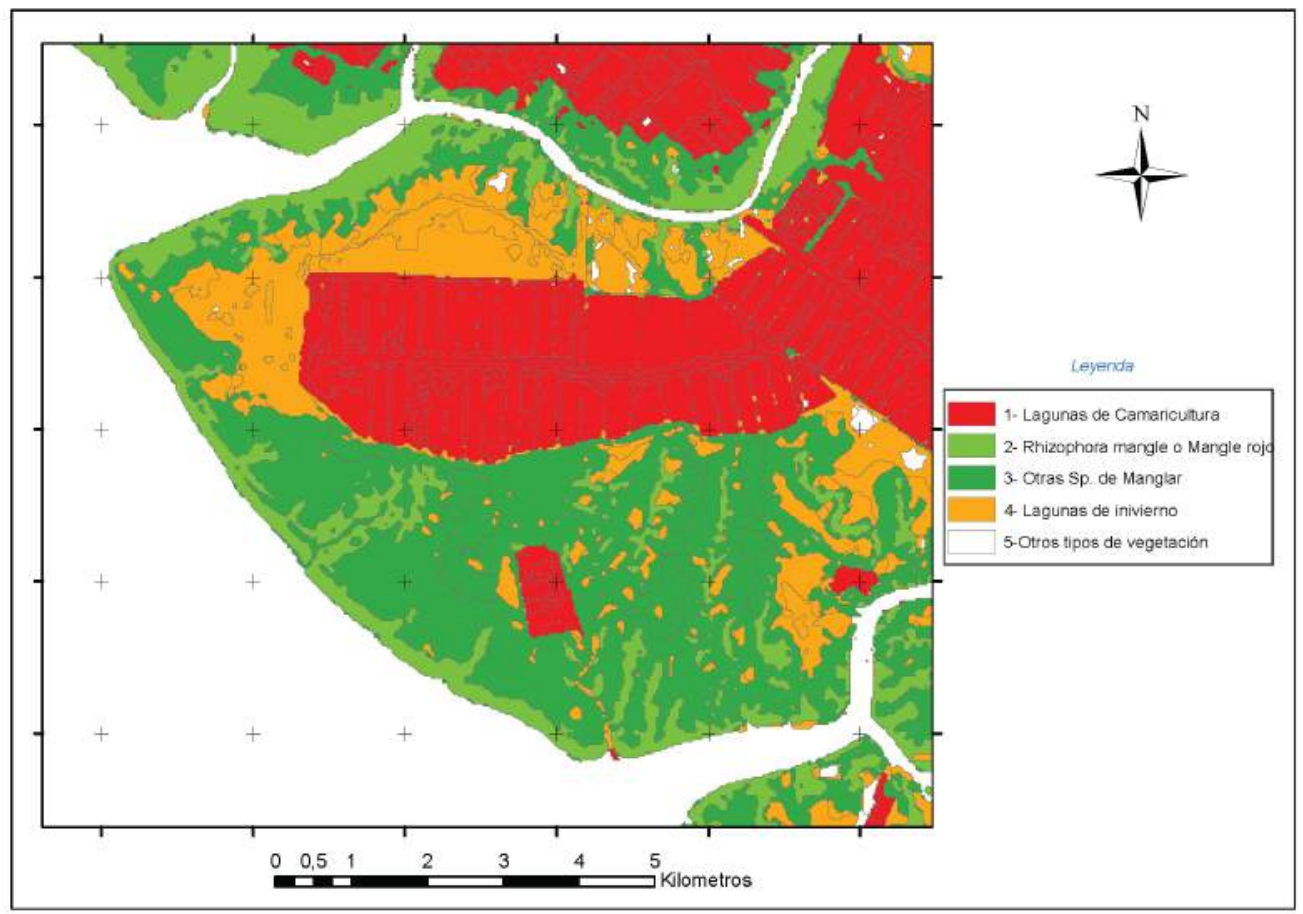

6. Validación de la clasificación: la correcta asignación de las categorías en el proceso de clasificación fue estimada con la ayuda de trabajo de campo realizado con la colaboración de representantes del Comité para la Defensa y Desarrollo de la Flora y Fauna del Golfo de Fonseca (CODDEFFAGOLF), técnicos de la organización española Enxeñería Sen Fronteiras (ESF) e investigadores de la Universidad Nacional Autónoma de Honduras (UNAH), lo que permitió conocer y corregir los errores cometidos en el proceso. 
7. Análisis de tendencias: los ficheros depurados resultantes de las clasificaciones calibradas y validadas fueron procesados con el programa $R$ ( $R$ Core Team, 2012) para analizar la tendencia seguida por las categorías en estudio y los cambios sucedidos entre las diferentes cubiertas a lo largo del período en estudio.

\section{RESULTADOS Y DISCUSIÓN}

Los resultados del proceso de clasificación de las imágenes satelitales permitieron verificar el avance de la industria camaronera, lo que ha dado lugar a un cambio significativo del ecosistema manglar y de otros humedales, ocasionando la pérdida de gran parte de su cobertura a lo largo del tiempo (ver cuadro 1).

En la figura 3 se puede apreciar la diferencia entre una clasificación de coberturas del suelo del año 1985 y otra de 2011, en la cual se observa la notable evolución de las lagunas naturales de camarón (categorías con tonos rojos y formas regulares).

El análisis de la serie muestra un aumento continuado, prácticamente lineal, de la superficie ocupada por las lagunas para cría de camarón en todo el golfo de Fonseca, desde escasamente 300 ha en 1985, hasta alrededor de 33,800 ha en 2011. Los resultados sugieren, por tanto, que el área ocupada por esta actividad se ha multiplicado por 100 en los últimos veinticinco años, a un ritmo de crecimiento estimado de casi 1,300 ha por año (ver figura 4).

La superficie ocupada por el bosque de manglar, por el contrario, muestra una tendencia decreciente, desde las 107,255 ha, calculadas en la imagen satelital clasificada de 1985, hasta las 79,633 ha de la imagen de 2011. Este descenso global del $25 \%$, parece haberse concentrado entre los años 1995 y 2000. Cabe resaltar que la dificultad para clasificar correctamente estas cubiertas, dada la alta probabilidad de confusión con otras coberturas existentes en la zona, resulta en un amplio intervalo de confianza (baja separabilidad espectral). 
Figura 3. Cubiertas identificadas en el golfo de Fonseca para los años 1985 (izquierda) y 2011 (derecha)

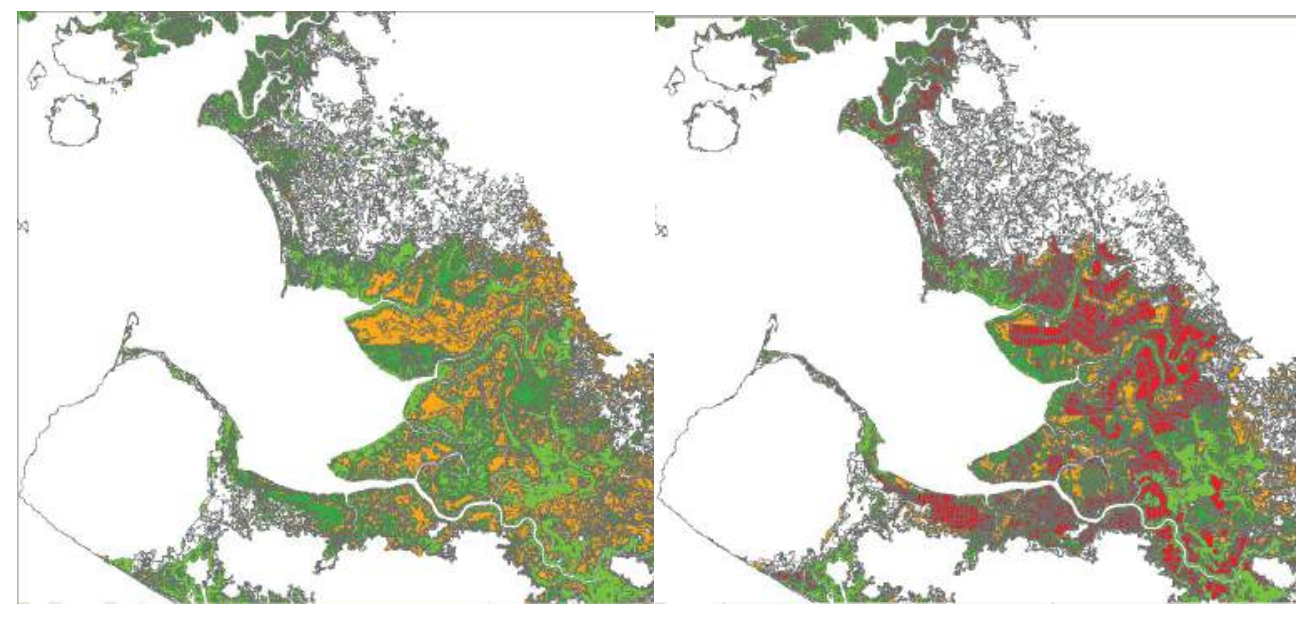

Cuadro 1. Evolución de la cubierta por año (manglar, camaronera)

\begin{tabular}{c|c|c|c|c|c} 
Año & $\begin{array}{c}\text { Área de manglar } \\
\text { (ha) }\end{array}$ & $\begin{array}{c}\text { Área de camaronera } \\
\text { (ha) }\end{array}$ & $\begin{array}{c}\text { Año } \\
\text { Área de manglar } \\
\text { (ha) }\end{array}$ & $\begin{array}{c}\text { Área de camaronera } \\
\text { (ha) }\end{array}$ \\
\hline 1985 & 107,255 & 297 & 1999 & 88,768 & 22,384 \\
1986 & 106,920 & 1,114 & 2000 & 89,999 & 25,180 \\
1987 & 97,555 & 2,022 & 2001 & 64,432 & 27,278 \\
1988 & -- & -- & 2002 & -- & -- \\
1989 & 99,753 & 4,847 & 2003 & -- & -- \\
1990 & 102,619 & 5,237 & 2004 & -- & -- \\
1991 & 115,877 & 6,969 & 2005 & -- & -- \\
1992 & -- & -- & 2006 & -- & -- \\
1993 & 117,798 & 10,327 & 2007 & 118,000 & 31,337 \\
1994 & 107,352 & 11,487 & 2008 & -- & -- \\
1995 & -- & -- & 2009 & 94,894 & 36,167 \\
1996 & 106,024 & 14,762 & 2010 & 78,202 & 40,430 \\
1997 & 87,276 & 17,777 & 2011 & 79,633 & 33,791 \\
1998 & 104,363 & 16,795 & & -- & -- \\
\hline
\end{tabular}


Figura 4. Evolución de la superficie de lagunas de camarón y bosque de manglar

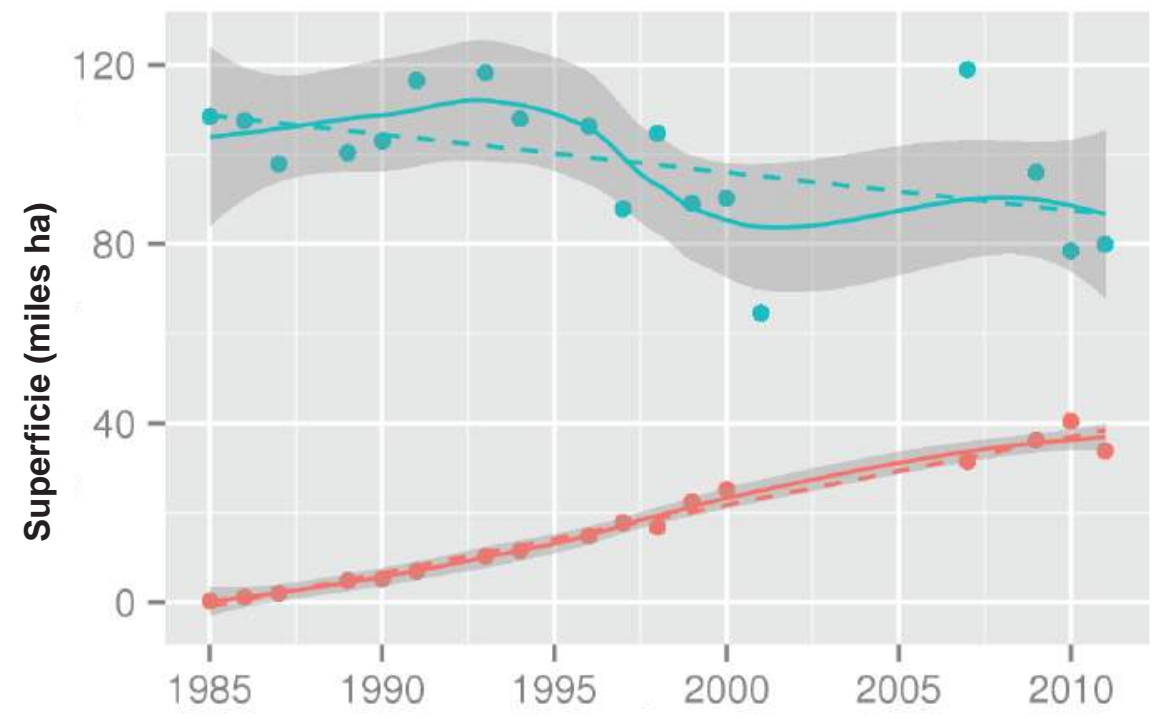

Años

Variable $\rightarrow$ Camaroneras $\rightarrow$ Manglar

El gráfico de la figura 4 muestra un ajuste de regresión local (línea continua) con intervalo de confianza al $95 \%$ y un ajuste lineal por mínimos cuadrados (línea de trazos). Alrededor de 12,207 ha de superficie ocupada por lagunas de cría de camarón en 2011 (un $36 \%$ del total en ese año) proceden de áreas clasificadas como manglar en 1985. El grueso de las áreas ocupadas por este proceso durante el período 1985-2011 vienen de láminas de agua de origen natural en 1985 (17,750 ha; el $54 \%$ de la superficie de camaroneras en 2011).

Analizado desde el punto de vista de la superficie de manglar, ello supone que alrededor del $44 \%$ de la superficie perdida en las áreas de manglar se debe a la ocupación, en última instancia, por lagunas de cría de camarón; mientras que el 56 \% restante se debió a la sustitución de cubiertas diferentes. DeWalt y otros (1996) determinaron que el mayor impacto sobre el bosque de manglar por lagunas para cría de camarón en Latinoamérica, corresponde a Ecuador y Honduras, en donde el descenso de mangle para Honduras (golfo de Fonseca) fue de un $22 \%$ entre el período de 1973 a 1992. Según la FAO (1996), la ocupación de lagunas artificiales de camarón en las áreas de manglares para 1988 eran de 28,213 ha con respecto a las calculadas en el presente estudio, que arrojan un dato de 16,795 ha; lo que indica 
que muchos estudios no excluyen las áreas de salineras, que en muchos casos alternan sus funciones con cría de camarón de forma artesanal.

\section{CONCLUSIONES}

De la comparación de cubiertas entre años consecutivos, se concluye que entre el 10-20 \% de la superficie ocupada por lagunas de camarón en cada año, procedía de áreas clasificadas como manglar en el año anterior. Por lo cual los resultados de las imágenes clasificadas ilustran lo que ha estado sucediendo en el golfo de Fonseca a lo largo de estos años: el avance de la expansión de las camaroneras ha provocado una disminución en gran parte de los manglares y humedales del Golfo de Fonseca, incluyendo espacios naturales protegidos.

Cabe mencionar que el ecosistema de manglar es un formador de nuevo suelo, por eso sus límites territoriales de línea de costa varían con el tiempo, lo que provoca que la dinámica de la costa sea alta, causando también cambios en las coberturas, por ello la especie de mayor fragilidad es el mangle rojo (Rhryzophora mangle L.); por lo que en la actualidad se está presentando un cambio natural de otras especies del sistema manglar como mangle negro (Avicennia germinans L.) y mangle blanco (Laguncuraria racemosa L.) especies que se encuentran en suelos más firmes y menos salados, mismos que por considerarse bosque secundario en el ecosistema de manglar, son menos protegidos y al degradarse terminan siendo alterados para la implementación de la camaricultura, con la presencia de lagunas artificiales.

\section{REFERENCIAS}

Alonso-Pérez, F.; Ruiz-Luna, A.; Turner, J.; Berlanga-Robles, C. y Mitchelson-Jacob, G. (2003). Land cover changes and impact of shrimp aquaculture on the landscape in the Ceuta coastal lagoon system, Sinaloa, México. Ocean \& Coastal Management, 46, 583-600.

Berlanga-Robles, C.; Ruiz-Luna, A.; Bocco, G. y Vekerdy, Z. (2011). Spatial analysis of the impact of shrimp culture on the coastal wetlands on the Northern coast of Sinaloa, Mexico. Ocean \& Coastal Management, 54, 535-543.

Bouman, C. y Shapiro, M. (1994). A multiscale random field model for bayesian image segmentation. IEEE Trans. on Image Processing, 3(2), 162-177. 
Chuvieco, E. (2008). Teledetección ambiental. La observación de la Tierra desde el espacio. Barcelona: Ariel.

FAO. (2005). Estudio de tendencias y perspectivas del sector forestal en América Latina. Informe Nacional Honduras. Recuperado de: http://www.fao.org

FAO. (2000). Cambios en la cobertura forestal, Honduras.Recuperado de: http://www.fao.org

FAO. (1996). Informe nacional para la conferencia técnica internacional de la FAO sobre los recursos fitogenéticos. Recuperado de: http://www.fao.org

GRASS Development Team. (2012). Geographic Resources Analysis Support System (GRASS) Software, Version 6.4.2. Open Source Geospatial Foundation. Recuperado de: http://grass.osgeo.org

Jarvis, A.; Reuter, H.I.; Nelson, A. y Guevara, E. (2008). Hole-filled SRTM for the globe version 4. CGIAR-CSI SRTM 90m Database. Recuperado de: http://srtm.csi.cgiar.org

R Core Team. (2012). R: A language and environment for statistical computing. $R$ Foundation for Statistical Computing. Austria. Recuperado de: http://www.R-project.org

Riaño, D.; Salas, J. y Cuvieco, E. (2000). Corrección atmosférica y topográfica, información multitemporal y auxiliar aplicadas a la cartografía de modelos de combustibles con imágenes Landsat-TM. Asociación de Geógrafos Españoles. Recuperado de: http://age.ieg.csic.es/metodos/docs/IX_2/Riano_David.PDF

Schowengerdt, R.A. (2007). Remote sensing. Models and methods for image processing. Burlington: Academic Press.

The Ramsar Convention. (2014). Recuperado de: http://www.ra-msar.org/country -profiles 